\title{
Oxalate-loaded microcapsule prepared with acrylonitrile-styrene copolymer (AS)
}

\author{
Fanbin Meng ${ }^{1, a}$,Shujun Wang ${ }^{1, b}$, Zhoupeng Duan $^{1, \mathrm{c}}$, and Xianliang Huo ${ }^{1, \mathrm{~d}}$ \\ ${ }^{1}$ State key laboratory of heavy oil processing, China University of Petroleum (Beijing), Beijing \\ 102249, China; \\ amfb1469@163.com, ${ }^{b}$ bjwsjbi@sina.com, ${ }^{c}$ syhgdzp@163.com, ${ }^{d}$ xianliang_huo@163.com
}

Keywords: microcapsule, oxalate, fracturing, acrylonitrile-styrene copolymer (AS)

\begin{abstract}
Oxalate-loaded microcapsules can be used as applicablecatalyst ofin-situ heat system for hydraulic gel fracturing. In this study, oxalate-loaded microcapsulewas prepared with AS as the shell material via phase separation. The product was characterized by thermogravimetric analyzer (TGA), laser diffraction particle size analyzer, and in vitro-release experiment. The test results indicate that the product obtained was particles composed of oxalate and acrylonitrile-styrene copolymer (AS) with the average particle size of $122.0 \mu \mathrm{m}$ and sustained-release performance.
\end{abstract}

\section{Introduction}

Microencapsulation is a technique packagingsolid,liquid or gaseous active ingredientsas the core material with shellmaterial to shield the active ingredient from the surrounding environment [1].As a technique to protect active ingredients from oxidation caused by heat, light, moisture, fromcontact with other substances over a long shelf life, to preventevaporation of volatile compounds and to control the release rate,microencapsulation has attracted researchers' great interests, and has been widely employed in the fields from chemicals and pharmaceuticals to cosmetics and printing such as, carbonless paper,scratch and sniff fragrance sampling, intelligent textiles, controlled release of drugs, pesticides and cosmetic active agents[2-5].

As microencapsulation technology can give the active ingredients (core material) sustained-release performance, oxalate-loaded microcapsules were introduced to the hydrofracturefor low temperature shallow reservoirs [6]. In the research of Wu etc. [7],the oxalate-loaded microcapsule wasprepared with ethyl cellulose (EC) as a shell material via phase separation in organic system, and get good sustained-release performance. However, the oxalate-loaded microcapsule prepared with acrylonitrile-styrene copolymer (AS) has not been reported.

The aim of this research is to prepared oxalate-loaded microcapsule with AS as the shell material via phase separation. The product obtainedwasparticles composed of oxalate and AS with theaverage particle size of $122.0 \mu$ mand sustained-release performance.

\section{Experimental}

\subsection{Materials}

Acrylonitrile-styrene copolymer (AS) was obtained from LG Chem., industrial grade. Oxalate $\left(\mathrm{H}_{2} \mathrm{C}_{2} \mathrm{O}_{4} \cdot 2 \mathrm{H}_{2} \mathrm{O}\right)$, n-heptane were supplied by Tianjin Guangfu Fine Chemical Research Institute, AR. Methylene dichloride (DCM), ammonium chloride $\left(\mathrm{NH}_{4} \mathrm{Cl}\right)$, and sodium nitrite $\left(\mathrm{NaNO}_{2}\right)$ were purchased from Beijing Chemical Plant, AR. Polydimethylsiloxanes (PDMS) were obtained from Dow Coring XIAMETER ${ }^{\circledR}$. Reagents all above were used as received.

\subsection{Synthetic procedures}

The synthesis procedures of the oxalate-loaded microcapsule shows as follow. The core material (oxalate, 1g) was added to the wall material (AS, $1 \mathrm{~g}$ ) DCM solution in a three-necked flask under stirring at the rate of $350 \mathrm{rpm}$. Coacervation of the AS was induced by dropwise addition of the PDMS (20 ml). After the dropwise, the mixture was quickly poured into a round-bottomed flask 
with condensing unit and $250 \mathrm{ml}$ of n-heptane under stirring at the rate of $450 \mathrm{rpm}$ for 20 minutes. Stop stirring for minutes to deposit the microcapsule. The obtained microcapsule was washed with n-heptane for two times, and dried at $60^{\circ} \mathrm{C}$ for three hours.

\subsection{Characterization}

Content testThe contents of product were investigated by a thermogravimetricanalyzer (TGA, HenvenHTG-3, China) with the range of $30-700{ }^{\circ} \mathrm{C}$ at a heating rate of $10^{\circ} \mathrm{C} / \mathrm{min}$ under a nitrogen atmosphere.

Particle size testsThe particle sizes of product were detected by the laser diffraction particle size analyzer (SALD-2300, SHIMADZU, Japan).

In vitro-release experiment Thesustained-release of the oxalate-loaded microcapsule was estimated as follows: $1.5 \mathrm{~g}$ microcapsule was added to the $100 \mathrm{ml}$ mixture of $\mathrm{NaNO}_{2}$ and $\mathrm{NH}_{4} \mathrm{Cl}$ aqueous solution with the mole fraction of $2.0 \mathrm{~mol} \cdot \mathrm{L}^{-1}$ at room temperature. And the reaction equation is described as follows [8]:

$$
\mathrm{NaNO}_{2}+\mathrm{NH}_{4} \mathrm{Cl} \stackrel{+}{\rightarrow} \mathrm{N}_{2} \uparrow+\mathrm{NaCl}+2 \mathrm{H}_{2} \mathrm{O}
$$

The values of the temperature of above system were collected by data recorder (TOPRIE, TP700, China) one time per second. For comparison, the catalytic performance of oxalate was estimated under identical conditions. It should be noted that the mass of oxalate directly added is equal to that loaded in the microcapsule.

\section{Result and discussion}

\subsection{Content}

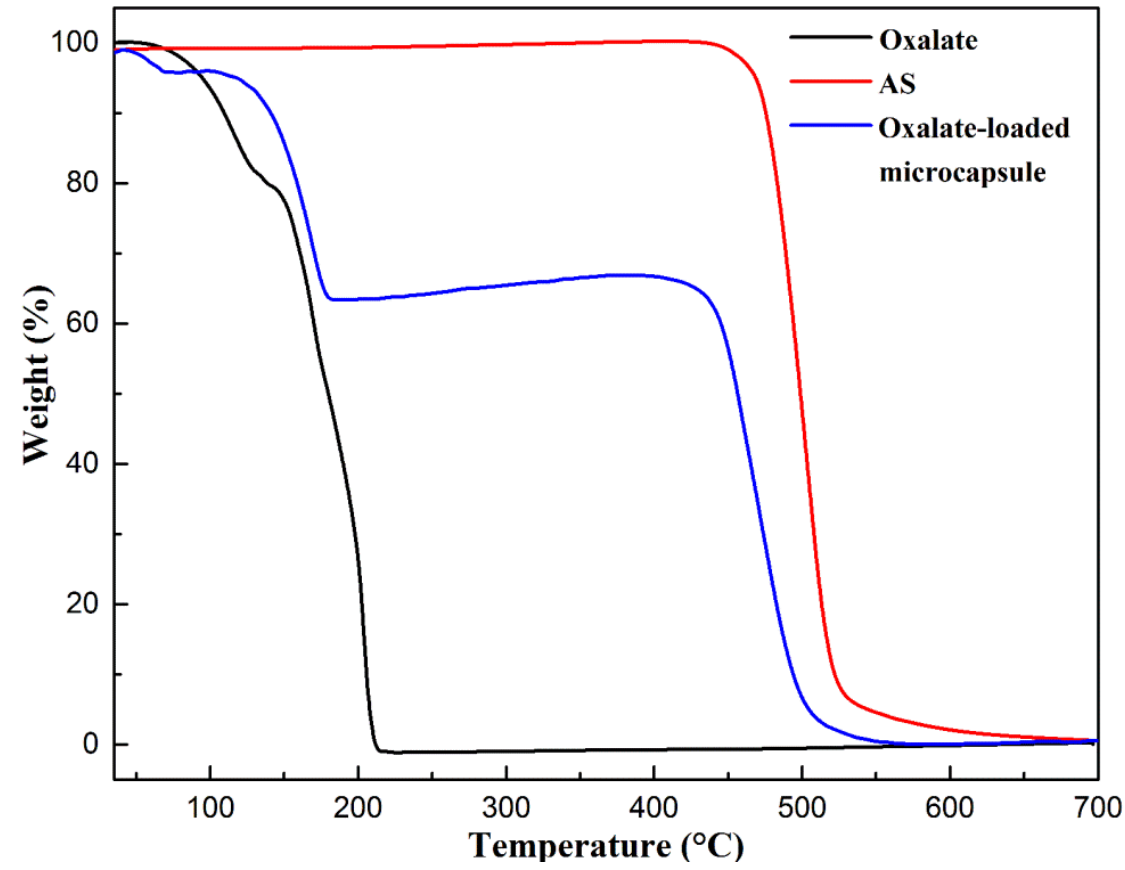

Fig. 1 The thermogravimetric weight loss (TG) curve of oxalate, AS, and the oxalate-loaded microcapsule

As we can see from the TG curve of oxalate-loaded microcapsule (Fig. 1), the product only occur decomposition during the decomposition temperature range of oxalate and AS. This thermal behaviour of the oxalate-loaded microcapsule indicates that the product is composed of oxalate and AS, and we can also know that the oxalate content of the product is 37.5 (wt) \%.

\subsection{Particle size}

The particle size distributions of sample prepared with AS via phase separation is showed in Fig. 2.The particle sizes of samples are micron-sized, and the average particle size is $122.0 \mu \mathrm{m}$. 


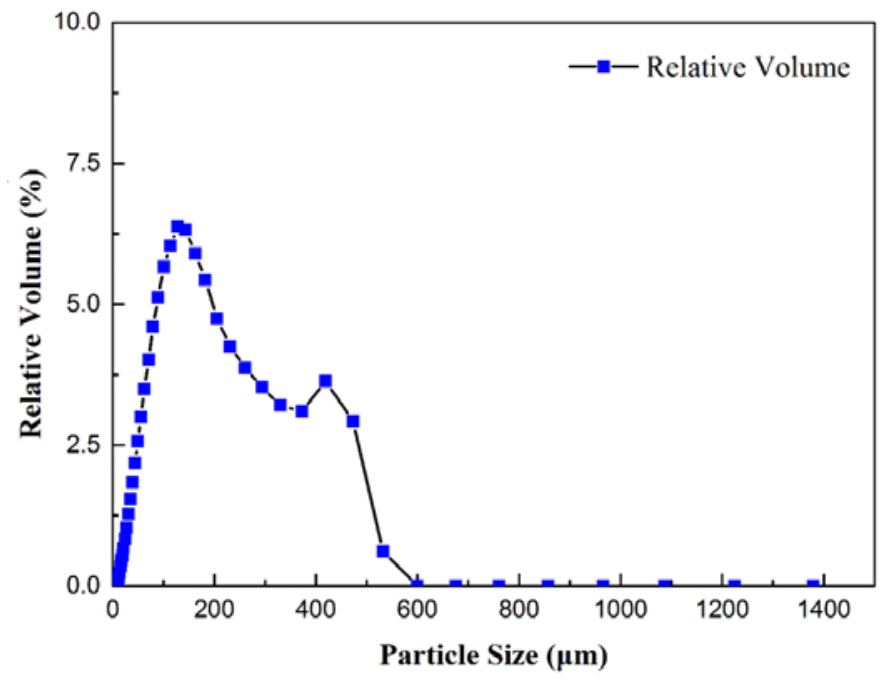

Fig. 2 Particle size distributions of the oxalate-loaded microcapsule

\subsection{Sustained-release performance}

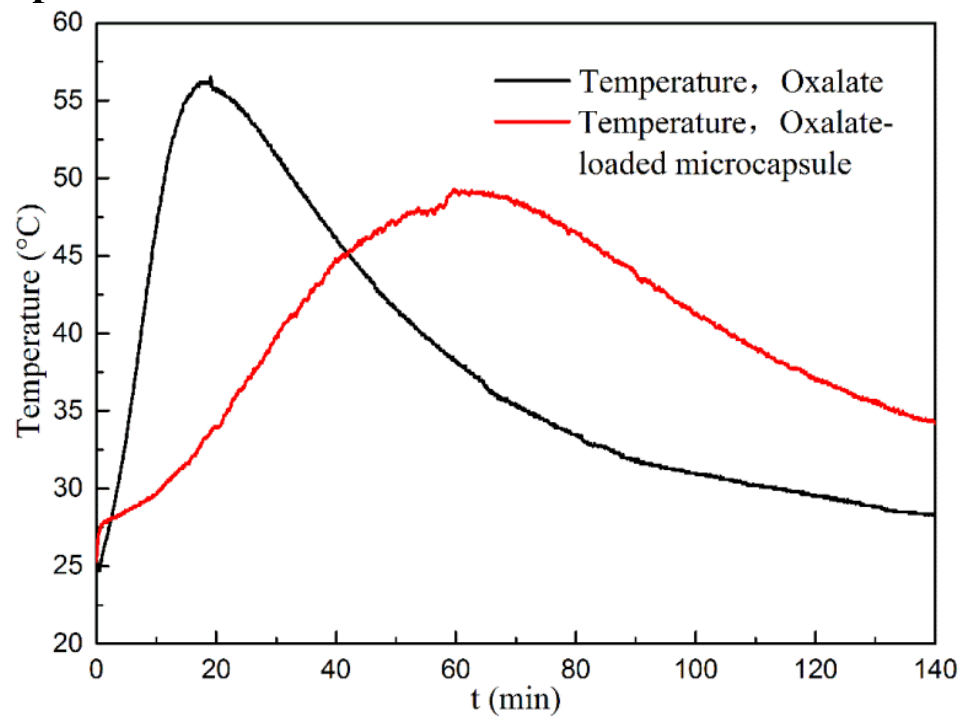

Fig. 3 Temperature of the exothermic reactioncatalysed with oxalate or oxalate-loaded microcapsule

Fig.3 shows catalytic performances of oxalate and oxalate-loaded microcapsule. Obviously, the system catalysed by oxalate-loaded microcapsule possesses longer time to reach the temperature peak.This finding leads to a conclusion that oxalate-loaded microcapsule possesses sustained-releaseperformance.

\section{Conclusion}

The oxalate-loaded microcapsule was prepared with AS via phase separation successfully. The product is micron-sized particles with the average particle size of $122.0 \mu \mathrm{m}$, the oxalate content of 37.5 (wt) \%, and sustained-release performance. The prepared oxalate-loaded microcapsule is a potentialcatalyst ofin-situ heat system for hydraulic gel fracturing.

\section{References}

[1] RamaDubey, T.C.Shami et al. Microencapsulation Technology and Applications. Defence Science Journal.Vol.59 (2009), p. 82-95.

[2] Isabel M. Martins, Maria F. Barreiro, et al. Microencapsulation of essential oils with biodegradable polymericcarriers for cosmetic applications. Chemical Engineering Journal.Vol.245 (2014),p. 191-200. 
[3] S.K. Ghosh.Functional coatings and microencapsulation: a general perspective. Functional Coatings. Wiley-VCH VerlagGmbH\&Co. KGaA, 2006, p. 1-28.

[4] S.O. Lumsdon, T.E. Friedmann, and J.H. Green. Encapsulationof oils by coacervation. WIPOPatent WO2005105290,2005.

[5] J.J.G.V. Soest. Encapsulation of fragrances and flavours: a way to control odour and aroma in consumer products.Flavours and Fragrances-Chemistry. Bioprocessing and Sustainability.Springer Germany, 2007, p. 439.

[6] M Cleary. Modelling and development of hydraulic fracturing technology. Vol. 275(1983),p. 383-475.

[7] W Jinqiao and Z Ning-sheng. Fracturing fluid with microencapsulated heat-generating system and corresponding technology. ACTA PETROLEI SINICA . Vol.26 (2005),p. 115-122.

[8] J.P. Ashton, L.J. Kirspel, and H.T. Nguyen. In-situ heat system stimulates paraffinic-crude producers in Gulf of Mexico. Society of Petroleum Engineers. 1989,p. 157-160. 